\title{
Dyspnea: when the preliminary imaging is unconvincing
}

\author{
Angelica Moretti, Francesca Croci, Franco Carmassi \\ Emergency Medicine, University of Pisa, Pisa, Italy
}

\begin{abstract}
A 73-year-old man was admitted to the Emergency Room (ER) for dyspnea and cough from several months. In ER were performed blood sampling, chest X-ray, electrocardiogram, echocardiogram and arterial blood gas. A thoracic ultrasound (US) revealed in the left side an abundant pleural effusion and a lung consolidation area of about $5 \mathrm{~cm}$ without air bronchogram. A thoracentesis showed the presence of hemorrhagic effusion. Chest computed tomography (CT) revealed micro-pulmonary embolism, abundant left pleural effusion with atelectasis of the lower ipsilateral lobe. Meanwhile the chest CT revised by the pulmonologist appeared suspicious for the presence of cancer, the cytological examination of pleural fluid revealed the presence of an adenocarcinoma. While the patient was waiting for the bronchoscopy he had a stroke and died in a few days. In conclusion, we believe that thoracic US has to be considered an extension of the physical examination, it is a bedside tool and it represents a valid diagnostic and therapeutic method. Therefore thoracic US, if closely linked to the physician's activity, can directly affect the decision-making process and management of the patient with dyspnea.
\end{abstract}

\section{Introduction}

Many patients come to Emergency Room (ER) for dyspnea and most of them are admitted to medical wards to study the cause of dyspnea. The present case helped us to assess the role and usefulness of thoracic ultrasound (US) in the differential diagnosis of dyspnea.

\section{Case Report}

A 73-year-old man was admitted to ER because of progressive dyspnea associated with coughing which had been present for several months. Since June 2012

Correspondence: Angelica Moretti, viale Castracani 243, 55100 Arancio (LU), Italy.

Mobile: +39.347 .7771185 .

E-mail: angelicamoretti@libero.it

Key words: Dyspnea; thoracic ultrasound.

Conflict of interest: the authors declare no conflict of interest.

See online Appendix for Figures.

Received for publication: 21 November 2013.

Revision received: 30 May 2014.

Accepted for publication: 1 July 2014.

This work is licensed under a Creative Commons Attribution NonCommercial 3.0 License (CC BY-NC 3.0).

(C) Copyright A. Moretti et al., 2015

Licensee PAGEPress, Italy

Italian Journal of Medicine 2015; 9:163-168

doi:10.4081/itjm.2015.451 he complained of a non-productive cough; in August the chest X-ray showed signs of emphysema, bilateral hilar enlargement, blunting of the left costophrenic angle. In early September 2012 he had progressive dyspnea, before at exercise and then at rest, associated with cough; therefore on September 28 he was admitted to ER.

The physical examination revealed that the patient was alert and oriented; he showed Glasgow coma scale 15 , normal cardiac rhythm, thoracic sounds diffusely reduced, especially on the left basal side, normal abdomen, peripheral pitting edema. Blood pressure (BP) was $150 / 80 \mathrm{mmHg}$, heart rate (HR) 99/min, respiratory rate (RR) $20 / \mathrm{min}, \mathrm{SO}_{2} 94 \%$, body temperature (BT) $36^{\circ} \mathrm{C}$.

His past medical history showed he was a former smoker; he had chronic obstructive pulmonary disease (COPD), pulmonary silicosis, hypertension, previous cerebrovascular accident and left eye hemianopia; he was treated with ACE inhibitors, calcium channel blocker and acetylsalicylic acid.

In ER the following exams had been performed: i) arterial blood gas (ABG): $\mathrm{pH}$ 7.416, $\mathrm{PaO}_{2} 62.8$ $\mathrm{mmHg}, \mathrm{PaCO}_{2} 44.7 \mathrm{mmHg}, \mathrm{HCO}_{3}-28.1 \mathrm{mmol} / \mathrm{L}$, $\mathrm{SO}_{2} 94.1 \%$; ii) electrocardiogram (ECG): sinus tachycardia; iii) chest X-ray: pleural left effusion, with adjacent parenchymal atelectasis; bilateral hilar enlargement; iv) blood tests: platelets (PLT) $102,000 / \mathrm{mcL}$, white blood cells (WBC) $11,310 / \mathrm{mcL}$ (N 76.9\%), creatinine $0.84 \mathrm{mg} / \mathrm{dL}$, international normalized ratio (INR) 1.2, activated partial thromboplastin time (aPTT) $28 \mathrm{~s}$, high-sensitivity (HS) troponin $91 \mathrm{ng} / \mathrm{L}$ (normal range: HS troponin $<14 \mathrm{ng} / \mathrm{L},<60$ $\mathrm{ng} / \mathrm{L}$ in elderly), C-reactive protein (CRP) $2.0 \mathrm{mg} / \mathrm{dL}$; v) monitoring of cardiac enzymes: negative; vi) as- 
sessment of clinical criteria predictive of pulmonary embolism: intermediate probability (age, HR $>95$ ) on Geneva score and low probability on Wells score; vii) intravenous therapy with furosemide and levofloxacin and oxygen therapy were administered.

Then the patient was then admitted to our ward.

\section{Formulation of the clinical problem}

Shortness of breath, persistent dry cough associated with radiological findings of pleural effusion and laboratory of neutrophil leukocytosis, elevated inflammatory markers in a patients with pulmonary silicosis, hypertension, previous smoking habits, previous cerebral ischemic attack.

In our department we repeated the physical examination, which is similar to ER examination. BP was $145 / 77 \mathrm{mmHg}$, HR $105 / \mathrm{min}$, RR $18 / \mathrm{min}$, BT $36.2^{\circ} \mathrm{C}$; we administered intravenous therapy with furosemide, piperacillin/tazobactam, methylprednisolone and transdermal nitrates, sodium dalteparin, aerosol therapy with corticosteroids, mucolytics and oxygen at $1 \mathrm{~L} / \mathrm{min}$.

\section{Formulation of diagnostic hypotheses}

Possible causes of dyspnea: i) acute coronary syndrome; ii) heart failure/acute pulmonary edema (APE); iii) acute respiratory distress syndrome (ARDS); iv) pulmonary embolism; v) COPD/asthma; vi) pulmonary inflammation; vii) lung cancer; viii) pneumothorax (PNX); ix) pleural effusion.

\section{Hypothesis testing}

Biochemical tests: creatinine $1.05 \mathrm{mg} / \mathrm{dL}$, blood urea nitrogen $41 \mathrm{mg} / \mathrm{dL}$, aspartate transaminase 22 $\mathrm{U} / \mathrm{L}$, alanine transaminase $17 \mathrm{U} / \mathrm{L}$, gamma-glutamyl transferase $25 \mathrm{U} / \mathrm{L}$, total bilirubin $0.39 \mathrm{mg} / \mathrm{dL}$, amylase $62 \mathrm{U} / \mathrm{L}$, lipase $30 \mathrm{U} / \mathrm{L}, \mathrm{Na} 141 \mathrm{mEq} / \mathrm{L}, \mathrm{K} 4.1$ $\mathrm{mEq} / \mathrm{L}$, brain natriuretic peptide (BNP) $24 \mathrm{pg} / \mathrm{mL}$, HS troponin $38 \mathrm{ng} / \mathrm{L}$, CRP $1.8 \mathrm{mg} / \mathrm{dL}$, hemoglobin $14.3 \mathrm{~g} / \mathrm{dL}$, PLT $115,000 / \mathrm{mcL}$, WBC $10,410 / \mathrm{mcL}$ (N 75\%), INR 1.2, aPTT $32 \mathrm{~s}$, fibrinogen $296 \mathrm{mg} / \mathrm{dL}$, AT III 89\%, D-dimer $6.3 \mathrm{mg} / \mathrm{L}$ (normal ranges: Ddimer $<0.50 \mathrm{mg} / \mathrm{L}$; HS troponin $<14 \mathrm{ng} / \mathrm{L},<60 \mathrm{ng} / \mathrm{L}$ in elderly).

$A B G\left(\mathrm{O}_{2}\right.$ at $\left.1 \mathrm{~L} / \mathrm{min}\right): \mathrm{pH} 7.406, \mathrm{PaO}_{2} 83.5 \mathrm{mmHg}$, $\mathrm{PaCO}_{2} 51.3 \mathrm{mmHg}, \mathrm{HCO}_{3}-31.5 \mathrm{mmol} / \mathrm{L}, \mathrm{SO}_{2} 97.0 \%$.

$E C G$ : sinus tachicardia, some ventricular extrasystoles.

Echocardiogram: no areas of hypokinesia (ejection fraction: 58\%), right sections within the limits.

Thoracic US performed in our department: on the left there is evident an abundant pleural effusion with a lung consolidation area of about $49 \times 43 \mathrm{~mm}$ without air bronchograms, of dubious nature. On the right there is no pleural effusion and no evident alteration of the parenchyma (Appendix Figures 1-6).

\section{Background query 1}

A history of COPD: how to assess the presence of an exacerbation? ${ }^{1}$

i) $A B G: \mathrm{PaO}_{2}<8.0 \mathrm{kPa}(60 \mathrm{mmHg})$ with or without $\mathrm{PaCO}_{2}>6.7 \mathrm{kPa}(50 \mathrm{mmHg})$; ii) chest $X$-ray: useful in excluding alternative diagnoses; iii) $E C G$ : useful in the diagnosis of co-existing heart disease; iv) spirometry: not recommended during a flare-up, because at this stage it may be difficult to perform and the measurements may be not accurate.

\section{Background query 2}

A history of pulmonary silicosis: what are the possible complications? ${ }^{2}$

The evolution is more or less rapid, depending on the amount of dust inhaled, and the nodular fibrosis may progress even after cessation of exposure, with the confluence of lesions and the formation of irregular masses typical of progressive massive fibrosis.

The complications and the most frequent causes of death are: i) tuberculosis (evidence of a pulmonary infiltrate on chest $\mathrm{X}$-ray); ii) respiratory failure; iii) right heart failure.

\section{Background query 3}

What are most frequent causes of ipsilateral pleural effusion?

i) liver cirrhosis (5\%): it occurs due to passage of fluid from the peritoneal space in the pleural cavity through defects in the diaphragm or lymphatic vessels, it is generally on the right side and it is a transudate; ii) pulmonary embolism (30-50\%): it occurs due to increase in the permeability of the visceral pleura in contact with the infarcted lung, it is usually an exudate, often with blood; iii) bacterial pneumonia (40\%): inflammation occurs in contact with the visceral pleura pneumonia; it is usually an exudate; iv) malignancies (pleural mesothelioma, lung or breast metastases) (>60\%): it occurs due to obstruction of the lymphatic vessels by neoplastic pleural implants, it is an exudate or frankly bloody, usually massive. The differential diagnosis is made by cytological examination.

\section{Background query 4}

Is there an association between silicosis and lung cancer?

There have been various studies on the association between silicosis and lung cancer which have given conflicting results. Also in terms of the concomitant presence of risk factors such as cigarette smoking: in some studies a close correlation between the two diseases has been demonstrated, ${ }^{3}$ while in others no correlation was found. ${ }^{4}$ 


\section{Summary}

In summary our case study was characterized by normal blood tests, cardiac enzymes, liver function tests and BNP, elevated D-dimer level; thoracic US with left pleural effusion, associated with atelectasis and parenchymal consolidation; non-diagnostic ECG and $\mathrm{ABG}$.

So the following were performed: i) thoracentesis: presence of hemorrhage $\rightarrow$ samples sent for cytological and cultural examination; ii) chest radiography (performed after a few days of antibiotic therapy): essentially unchanged; iii) thoracic CT with and without contrast medium: some lymphadenopathy of significant size, small filling defects related to micro-pulmonary embolism to the left lung, abundant left pleural effusion with atelectasis of the lower ipsilateral lobe. No clear images related to focal parenchymal lung segments disease; iv) pulmonary scintigraphy with Tc99: findings of pulmonary embolism.

\section{Review of the case}

A contrast-enhanced chest CT was performed for further evaluation of the area of parenchymal consolidation highlighted by thoracic US, which appeared suspicious for cancer. The diagnosis of pulmonary embolism was occasional, as ECG and ABG were not highly suggestive and high $\mathrm{D}$-dimer and clinical symptoms (dyspnea and fatigue) could be attributed to the presence of abundant pleural effusion.

As the response of the chest CT was unconvincing, especially for the presence of parenchimal consolidation detected by thoracic US, we did ask the pneumologist to review the chest CT.

When the chest CT was revised by the pulmonologist it appeared suspected for cancer of left lung (consolidation highlighted with an echo?).

Therefore the following tumor markers were also assayed: Ca125 3089 U/mL, Ca15.3 1044 U/mL, carcinoembryonic antigen $584.3 \mathrm{ng} / \mathrm{mL}, \beta 2$-microglobulin $2420 \mathrm{mcg} / \mathrm{L}$, neuron specific enolase $28 \mathrm{ng} / \mathrm{mL}$, Cyfra $21.1200 \mathrm{ng} / \mathrm{mL}$. Within the limits $\alpha$-fetoprotein, free and total prostate specific antigen, Ca19.9, ferritin.

Then the results of pleural fluid examination became available: i) cytological examination of pleural fluid: adenocarcinoma; ii) sputum culture of pleural fluid: negative for aerobes, anaerobes and fungi.

Then, we made further investigations in order to stage the disease: i) US abdomen: inhomogeneous liver parenchyma, bilateral renal cysts, prostate enlargement, absence of abdominal effusion; ii) CT abdomen with and without contrast medium: suspected secondary liver lesions. Slightly enlarged left adrenal gland. Slightly enlarged retroperitoneal lymph nodes. Renal cysts. Diverticula of the colon. Aereobilia.

\section{Evolution of the case}

While waiting for the bronchoscopy, the patient was confused and dysphoric.

Brain CT without contrast: hypodense lesion extended cortico-subcortical temporo-occipital right, compatible with ischemic injury in the subacute phase with small intracerebral hemorrhages.

Neurological consulting: clonazepam and dexamethasone added to the pharmacological therapy.

Brain CT without contrast medium after $48 \mathrm{~h}$ : unchanged.

Consulting oncology: the clinical picture determined by comorbidities (pulmonary embolism and stroke) does not allow to complete the diagnostic/stadiation process.

During the following days the patient alternated between phases of psycho/motor agitation and stupor, resulting in death on the eighth day from stroke due to respiratory failure.

\section{Discussion}

\section{Studies: path finding of ultrasound in the event of dyspnea}

Devices and transducers: The high-frequency linear probes give information on the most superficial structures, as well as on the morphology of the pleural line and its scroll mode; the low-frequency convex probes allow a deeper view, but they are less defined on the superficial layers, therefore they are more useful for the study of pleural effusion and thickening; the sectoral probes, cardiology-type, have characteristics similar to the convex probes, but have the advantage of being easily positioned in the intercostal spaces.,

Thoracic projections: The study of the chest can be performed on the patient in a sitting or supine position, using longitudinal or transverse scans (actually oblique along the intercostal spaces).

The comprehensive examination includes an extensive study of both lungs, both front and rear, from the apex to the base, on longitudinal parallel lines, from midsternal/posterior median line to axillary line. ${ }^{5}$

\section{Semeiotics of thoracic ultrasound}

Identification of the pleural line and its morphology (Appendix Figure 7): The pleural line appears as a thin hyperechoic line which, in the longitudinal scans, is located immediately below the costal plane, and in the transverse/oblique scans, immediately below the plane of the soft tissues. ${ }^{5,7,8}$

Type pleural interface (Appendix Figure 8): US image of a normal lung looks completely artefactual as a result of the interaction of US with the air interface generated by the gases contained in the ventilated lung. 5,7,8 
Evaluation of the gliding sign (Appendix Figures 9 and 10): Physiological flow of the pleural line (sliding or gliding sign), synchronous with the respiratory excursions, is most evident at the lung bases and less at the apex. The absence of gliding sign is always pathological. The lung point is the break point beyond which the physiological movement of sliding of the pleural line is no longer perceptible. ${ }^{5,8}$

Type of rear artifacts (Appendix Figures 11 and 12): Lines $A$ : horizontal artifacts that are generated by a reverb mechanism linked to the ample variation in acoustic impedance between the soft tissues of the chest wall and the air contained in the lung; they are parallel to the pleural line, equidistant from each other as is the probe from the pleural line, of decreasing intensity from the surface into the depth., 5,89

Lines $B$ : vertical artifacts extending in depth without weakening the entire length of the US scan. Their presence is an expression of the increase in extravascular lung water and their number correlates with the extent of the interstitial involvement. A number of lines $B$ up to 3 for scan US is of no clinical importance, but when their number increases they can contribute to create a confluent framework, called white lung., ${ }^{5,8,10}$

\section{Evaluation of dyspnea with thoracic ultrasound}

Dyspnea in acute coronary syndrome: Normally dyspnea is associated with retrosternal chest pain. ECG, monitoring of cardiac enzymes and cardiac US are useful in the diagnosis.

Thoracic US: non-diagnostic.

Dyspnea in heart failure/APE (Appendix Figures 13 and 14): In general, the dyspnea is associated with orthopnea and paroxysmal nocturnal dyspnea. ECG, BNP assay, chest X-ray and cardiac US are useful in the diagnosis.

Thoracic US: the usual configuration of reverberant horizontal lung (lines A) changes in a vertical pattern (comets lung), i.e., in pathological multiple lines $\mathrm{B}$, which can be separated by a distance which corresponds to that separating the normal interlobular septa $(0.7$ to $1.5 \mathrm{~cm}$ ) or confluent (white lung, which corresponds to areas of ground glass on chest CT) $\rightarrow$ wet lung. The extent of the lines B correlates with the degree of interstitial involvement. . $^{5,10,11}$

Dyspnea in ARDS (Appendix Figures 15 and 16): Typically acute dyspnea is caused by a group of acute lung injuries that recognize various etiologies (sepsis, pancreatitis, trauma, inhalation, etc.) and a common pathogenic mechanism (inflammatory). ABG, echocardiogram and chest X-ray are useful in the diagnosis.

Thoracic US: the pleural line is always abnormal (thickened and irregular), with lung pulse (the area of consolidated lung presents a motion-like vibration, unrelated to the breathing action but synchronous with cardiac activity) and reduction or abolition of lung sliding; an heterogeneous involvement of the lung is evident, which causes areas of ground glass with areas of consolidation, for which there is the presence of areas of savings..$^{5,12,13}$

Dyspnea in pulmonary embolism (Appendix Figure 17): In general, the dyspnea has an acute onset and may be associated with pleuritic pain, coughing and sometimes hemoptysis. ECG, ABG, D-dimer assay and contrast-enhanced chest CT may be useful in the diagnosis. Geneva score and Wells score are useful as predictive clinical criteria.

Thoracic US: normal findings (absence of lines B) $\rightarrow$ dry lung. ${ }^{5,14}$

Dyspnea in COPD/asthma (Appendix Figure 18): Dyspnea is usually chronic, associated with cough $\mathrm{ABG}$ and chest $\mathrm{X}$-ray may be useful in the diagnosis.

Thoracic US: no specific images, the subpleural plan may appear normal and in any case there are no vertical artifacts (lines B) $\rightarrow$ dry lung. ${ }^{5,15}$

Dyspnea in pulmonary inflammatory thickening (Appendix Figure 19): Dyspnea is usually associated with fever, productive coughing and chest pain. Chest $\mathrm{X}$-ray and blood tests (white blood cell count and inflammatory markers) are useful in the diagnosis.

Thoracic US: hypoechoic area of variable shape, with mixed salt and pepper texture, hyperechoic and/or reverberant striae representing the bronchioles containing air (air bronchograms), transonic tubular structures representing the vessels and tubular structures with no flow doppler sampling representing bronchi filled with fluid (fluid bronchograms). ${ }^{5,16-18}$

Dyspnea in cancer (Appendix Figure 20): Typically is characterized by progressive dyspnea and may be associated with pleuritic pain, cough and sometimes hemoptysis. Chest X-ray, contrast-enhanced chest CT, pleural fluid analysis and bronchoscopy are useful in the diagnosis.

Thoracic US: hypoechoic area with fairly homogeneous echogenicity, irregular polycyclic contours, without air bronchograms. ${ }^{5,19}$

Dyspnea in PNX (Appendix Figure 21): There is usually an acute onset, shortness of breath associated with pleuritic chest pain, which does not improve with the administration of oxygen. $\mathrm{ABG}$, the chest $\mathrm{X}$-ray and possibly the chest $\mathrm{CT}$ are useful in diagnosing.

Thoracic US: absence of the gliding sign, namely, non-sliding visceral pleura due to the presence of air in the pleural cavity, and the lung pulse, presence of lung point, namely the break point beyond which perceptible physiological movement scroll of the pleural line is no longer appreciable. . $^{50,21}$

Dyspnea in pleural effusion (Appendix Figure 22): Typically dyspnea has a progressive onset and can be associated with pleural pain and coughing. Chest Xray, chest CT, and analysis of the pleural fluid are useful in the diagnosis. 
Thoracic US: anechoic area bounded by the chest wall and diaphragm, mobile with breaths; it may appear as a transudate (transonic, liquid) or an exudate (corpusculated, with the presence of septa of fibrin, etc.). US is used to detect not only the presence, but also the extent and the nature of the pleural effusion, and then to evaluate the opportunity to proceed with a drainage. $5,20,22,23$

The flow-chart on the role of thoracic US in patients with dyspnea is reported in Appendix Figure 23.

\section{Conclusions}

In expert hands, thoracic US is accurate in diagnosing pleural effusion, PNX, infiltration of the pulmonaryalveolar-interstitial space (cardiogenic acute pulmonary edema and ARDS) and consolidation. Sometimes PNX cannot be seen on the chest radiography, especially when PNX is small and in the presence of massive pleural effusion, so lung US more accurately rules in the diagnosis of PNX than supine anterior chest radiography and correlates well with CT in the assessment of PNX extension. In the evaluation of an interstitial syndrome at thoracic US signs of the cardiogenic acute pulmonary edema are: pathological bilateral and multiple lines B, often confluent (white lung), pleural line thin, presence of lung sliding and, often, presence of pleural effusion. In ARDS, thoracic US unveil the anterior subpleural consolidations, absence or reduction of lung sliding, spared areas of normal parenchyma, pleural line abnormalities and nonhomogeneous distribution of B lines. Lung consolidation may have a variety of causes including lung cancer, compression atelectasis and obstructive atelectasis. Additional US signs which may help to determine the cause of lung consolidation include the quality of the deep margins of the consolidation, the presence of comet-tail reverberation artifacts at the far-field margin, the presence of air and/or fluid bronchograms and the vascular pattern within the consolidation. On thoracic US lung cancer usually appears as a hypo-echoic area with fairly homogeneous echogenicity, irregular polycyclic margins without air bronchograms, whereas in atelectasis the air bronchograms appear static and have a parallel course and comet-tail reverberation artifacts at the far-field margin are absent.

So, in our opinion, pleural and peripheral pulmonary lesions can be characterized by thoracic US. Although thoracic US is operator-dependent, it has a number of advantages over CT, including bedside approach, lower cost and no radiation exposure, better manipulation for severely ill patients and guiding lifesaving therapies in extreme emergency. ${ }^{8,24,25}$

\section{In summary}

The thoracic US: i) can be considered an extension of the physical examination; ii) it is a bedside method that can be repeated without discomfort for the patient; iii) it allows an easy follow-up without exposure to ionizing radiation; iv) it is non-invasive; v) it represents a valid diagnostic and therapeutic tool (USguided thoracentesis).

The integration of data that can be obtained quickly from US evaluation of the heart, lungs and inferior vena cava are fundamental for the diagnosis and treatment of patients with dyspnea.

Therefore thoracic US, can directly affect the decision-making process and management of the patient with dyspnea.

\section{References}

1. Global Initiative for Chronic Obstructive Lung Disease (GOLD). Global strategy for diagnosis, management and prevention of COPD; rev. 2012-2013. Available from: http://www.goldcopd.com

2. Leung CC, Tak Sun Yu I, Chen W. Silicosis. Lancet 2012;379:2008-18.

3. Rice FL, Park R, Stayner L, et al. Crystalline silica exposure and lung cancer mortality in diatomaceous earth industry workers: a quantitative risk assessmet. Occup Environ Med 2001;58:38-45.

4. Hnizdo E, Murray J, Klempman S. Lung cancer in relation to exposure to silica dust, silicosis and uranium production in South African gold miners. Thorax 1997; 52:271-5.

5. Soldati G, Copetti R. Ecografia toracica. Torino: C.G. Edizioni Medico Scientifiche; 2012.

6. Lichtenstein D. Ultrasound in the management of thoracic disease. Crit Care Med 2007;35:S250-61.

7. Mathis G. Thoraxsonography part II: chest wall and pleura. Ultrasound Med Biol 1997;23:1131-9.

8. Volpicelli G, Elbarbary M, Blaivas M, et al. International Liaison Committee on Lung ultrasound (ICCLUS). International evidence-based recommendations for point-of-care lung ultrasound. Intensive Care Med 2012;38:577-91.

9. Lichtenstein D. Lung ultrasound in the critically ill. In: Vincent J-L, ed. Year book of intensive care and emergency medicine. Berlin: Springer; 2004. pp 625-644.

10. Lichtenstein D, Mezière G, Biderman P et al. The comettail artifact: an ultrasound sign of alveolar-interstitial syndrome. Am J Respir Crit Care Med 1997;156:1640-6.

11. Picano E, Frassi F, Agriccola E, et al. Ultrasond lung comets: a clinically useful sign of extravascular lung water. J Am Soc Echocardiogr 2006;19:356-63.

12. Copetti R, Soldati G, Copetti P. Chest sonography: a useful tool to differentiate acute cardiogenic pulmonary edema froma acute respiratory distress syndrome. Cardiovasc Ultrasound 2008;6:16.

13. Arbelot C, Ferrari F, Bouhemad B, et al. Lung ultrasound in acute respiratory distress syndrome and acute lung injury. Curr Opin Crit Care 2008;14:70-4.

14. Mohn K, Quiot JJ, Nonent M, et al. Transthoracic sonography in the lung and pleura in view of a suspected pulmonary embolism. J Ultrasound Med 2003;22:673-8.

15. Lichtenstein D, Mezière G. A lung ultrasound sign al- 
lowing bedside distinction between pulmonary edema and COPD: the comet tail artifact. Intensive Care Med, 1998,24:1331-4.

16. Mathis G. Thoraxsonography part II: peripheral pulmonary consolidations. Ultrasound Med Biol 1997; 23:1141-53.

17. Parlamento S, Copetti R, Di Bartolomeo S. Evaluation of lung ultrasound for the diagnosis of pneumoniae in the ED. Am J Emerg Med 2009;27:379-84.

18. Cortellaro F, Colombo S, Coen D, et al. Lung ultrasound in an accurate diagnostic tool for the diagnosis of pneumoniae in the emergency department. Emerg Med J 2012;29:19-23.

19. De Gregorio MA, D’Agostino H. Ultrasound in pneumologo: the current situation. Arch Bronconeumol 2003; 39:535-6.

20. Sperandeo M, Filabozzi P, Varriale A, et al. Role of thoracic ultrasound in the assessment of pleural and pulmonary diseases. J Ultrasound 2008;11:39-46.

21. Lichtenstein D, Mezière G, Biderman P, Gepner $A$. The "lung point": an ultrasound sign specific to pneumothorax. Intensive Care Med 2000;26:1434-40.

22. Blackmore CC, Black WC, Dallas RV, Crow HC. Pleural fluid volume estimation. A chest radiograph prediction rule. Acad Radiol 1996;3:103-9.

23. Tsai TH, Yang PC. Ultrasound in the diagnosis and management of pleural disease. Curr Opin Pulm Med 2003; 9:282-90.

24. Ashton-Cleary DT. Is thoracic ultrasound a viable alternative to conventional imaging in the critical care setting? Br J Anaesthesia 2013;111:152-60.

25. Elhafez SAMA, Shehata MEA, Rashed MAE, et al. Echopulmonography versus computed tomographic chest predictors for differentiation between benign and malignant peripheral pulmonary and pleural lesions. Egypt J Chest Dis Tubercol 2013;62:127-35. 